
\title{
Filmpädagogik
}

* Zurück zur Übersicht über den

Themenschwerpunkt

\section{Film als Text im Unterricht. Show us a Story}

\section{CAREN WILLIG}

Während ich diesen Artikel schreibe, bearbeitet ein Kollege von mir einen Kurzfilm am Computer. Ich würde am liebsten sofort tauschen. Nicht, dass ich Schreiben nicht mag. Mir geht es dabei nur so wie vielen Schülern - ich habe mit Filmen und Filmarbeit schon immer mehr Spaß assoziiert als mit gedruckten Texten. Deutsch war mein Lieblingsfach, und obwohl ich außergewöhnlich gerne las und schrieb, ist eine meiner liebsten Erinnerungen die Produktion eines Kurzfilms im Deutschunterricht. Ich habe mich selten so intensiv mit dem Lehrmaterial und meinen Mitschüler/innen auseinandergesetzt und bin dankbar, dass immerhin eine Lehrerin Film ernst genommen hat. Schade war, dass unser Endprodukt aufgrund mangelnder Kenntnis von Filmkonventionen und Filmsprache nicht wirklich kinoreif war und dass die meisten von uns unser neu gewonnenes Wissen nie mehr im Unterricht anwenden konnten. Hätten wir Filmkompetenz (cineliteracy - wie es im Englischen treffend genannt wird) und die Ansicht, dass auch Medientexte es wert sind, studiert und analysiert zu werden, im Unterricht vermittelt bekommen, wäre ein filmsprachgewandter Kurzfilm sicher nur ein positives Resultat gewesen. Leider wussten die meisten Lehrer/innen den Impakt von Medien im Klassenzimmer nicht zu schätzen, und wenn sie doch mal Filme im Unterricht einsetzten, wurde ihnen Bequemlichkeit unterstellt. An der Universität war es leider nicht viel anders, und so musste ich mich einige Jahre gedulden, um wieder mit Film - wenn auch immer noch nicht über Film - zu lernen. In Literaturwissenschaft ist mir eine Vorlesung noch sehr gut in Erinnerung, da Ästhetik mit Hilfe von Musikvideos besprochen wurde, und eine Verfilmung von Goethes „Die Leiden des jungen Werther". Kein anderes Medium hat mir Goethe so nahe gebracht, die Charaktere so menschlich gemacht. Es war eine exzellente Verfilmung, basierend auf einem exzellenten Buch. Es geht nicht darum, Drucktexte abzutun oder zu verdrängen, sondern Filme als Texte (texts in their own right) zu erkennen.
Das ist genau, was wir in der Abteilung Education des British Film Institute (bfi) zu vermitteln versuchen. Es ist ein einfaches Konzept: Medien - Film, Fernsehen, Video und zunehmend das Internet und Computerspiele - machen einen wichtigen und wertvollen Teil unserer Kultur aus und sollten als Kulturgut anerkannt werden. Nur wenn diese Erkenntnis in das Konzept der Bildung einbezogen wird, wenn Medienkompetenz vermittelt wird, können wir Kindern und Jugendlichen helfen, sich in einer von Medien geprägten Welt zurechtfinden.

\section{Aufgaben der Medienpädagogik - media education}

Während es lange Zeit darum ging, Kinder und Jugendliche vor negativen Auswirkungen des Medienkonsums zu schützen, wird es im Zeitalter der wachsenden Bilder- und Informationsflut immer wichtiger, den kritischen und auch kreativen Umgang mit Medien zu vermitteln. Heranwachsenden sollen die Mittel an die Hand gegeben werden, Medien produktiv zu nutzen und medial vermittelte Inhalte kritisch zu hinterfragen. Gerade heutzutage, wo Medien allgegenwärtig sind, kann die Wichtigkeit der Medienpädagogik kaum überschätzt werden. Medien beeinflussen fast alle Aspekte unseres Alltages, und obwohl sie so transparent wirken können, sind sie oft schwer zu durchschauen. Um nicht von den Medien beherrscht zu werden und sich ihrer Einflüsse bewusst zu sein, müssen wir mehr mit und über Medien lernen. Dieses Wissen weiterzugeben, zu lehren, Inhalte und Ästhetiken der Medien $\mathrm{zu}$ verstehen und $\mathrm{zu}$ hinterfragen, und sie kreativ umzusetzen - also Medienkompetenz zu vermitteln -, ist Aufgabe der Medienpädagogik. Immer mehr Institutionen, und auch die Medienindustrien, erkennen den Stellenwert und fördern Medienpädagogik in Großbritannien, ein Beispiel dafür ist das British Film Institute.

Das British Film Institute (bfi) und moving image media education

Das bfi wurde 1933 gegründet, um die Entwicklung von Film-, Fernseh- und Videoproduktionen in Großbritannien $\mathrm{zu}$ fördern, und setzt sich seit 1950 für die 
Aufnahme der Medienpädagogik in das nationale Curriculum ein. Die Begriffe media education und "Medienpädagogik", umschreiben ein weites Feld von Tätigkeiten und Aktivitäten im Zusammenhang mit Medien, es gibt keine wirklich einheitliche Definition des Begriffs media education in England. Cary Bazalgette, Leiterin der Abteilung bfi Education, argumentiert, dass ,wenn irgend jemand versucht dogmatisch festzusetzen, was media education ist, er in Wirklichkeit nur offenbart, wie er media education gerne aufgefasst haben möchte". In bfi Education konzentriert man sich daher auf moving image media education und macht sich seit 20 Jahren dafür stark, dass dies in Bildungseinrichtungen ernst genommen wird. Die Tatsache, dass das bfi eine staatliche Organisation ist, hat geholfen, die englischen Erziehungsbehörden auf die Wichtigkeit von moving image media education aufmerksam zu machen und ihre Strategien zu beeinflussen. bfi Education setzt sich für fächerübergreifendes Lehren unter dem Begriff moving image media education ein und versucht Lehrer/innen mit Hilfe von Kursen und Konferenzen und der Veröffentlichung von anspruchsvollen Lehrmaterialien, nebst vieler anderer Aktivitäten, für moving image media education zu begeistern und ihnen Hilfsmittel für effektives Lehren mitzugeben.

\section{Medienpädagogik im englischen Curriculum}

Während es in den 80er Jahren an politischer Unterstützung medienpädagogischer Projekte mangelte, hat sich jetzt immerhin auch auf politischer Ebene die Einsicht durchgesetzt, dass Medienpädagogik ein wichtiger Bestandteil in der Ausbildung sein sollte. Dieses gewachsene politische Bewusstsein hat sich bisher jedoch noch nicht in langfristige finanzielle Unterstützung gewandelt, und es braucht noch viel lobbying, damit Medienpädagogik nicht nur von hoch motivierten Einzelpersonen unterrichtet wird. Oft wird moving image media education eher zufällig in den Hauptunterricht integriert, was die Qualität des Medienunterrichts extrem schwankend macht. Es gibt immer noch keine einheitlichen Kriterien, an denen man einen erfolgreichen Medienunterricht messen könnte. Die Vorgaben zum Thema Medienpädagogik sind folglich sehr allgemein gefasst. Doch Cary Bazalgette verteidigt diesen Zustand, da es ihrer Meinung nach in der momentanen Lage gar nicht möglich wäre, detailliertere Vorgaben aufzustellen: „Ich denke, daß es in einer Situation, in der die $\mathrm{R} e$ sourcen und vor allem die Modelle des Lernfortschritts nicht existieren, unverantwortlich ist, einem Englischlehrer ein ausgefeiltes medienpädagogisches Curriculum vorzuschreiben" (Bazalgette 1996). Bazalgette plädiert daher für intensive Forschung zu Me- thoden, Zielen und der Überprüfbarkeit vermittelter Erkenntnisse im Bereich Medienpädagogik, bevor es zu einer detaillierteren Festlegung medienpädagogischer Inhalte durch ein Curriculum kommen kann. Lehrer/innen, die motiviert sind im Unterricht mit Filmtexten zu arbeiten, lässt das jetzige Curriculum viele Möglichkeiten offen und zwingt sie nicht, nach strengen Richtlinien in einem ohnehin schon sehr vorgeschriebenden Lehrplan zu unterrichten. Jedoch können weniger interessierte Lehrer diese Möglichkeiten auch übergehen, was leider immer noch viel zu häufig der Fall ist.

\section{Medienpädagogik an englischen Schulen}

In der englischen National Literacy Strategy for primary school children gibt es noch keine Vorschriften, dass Kinder über Bildmedien lernen sollten. Medienpädagogik hat aber seit mehr als 20 Jahren an weiterführenden englischen Schulen einen Platz und hat sich als media studies in den höheren Klassen etabliert. Während auch in niedrigeren Klassen generell durchaus Interesse von Lehrer/innen da ist, Film und Fernsehen im Unterricht zu verwenden, würden die meisten Lehrer/innen sich schwer tun, ein anderes Fach wie Medienpädagogik zum Lehrplan hinzuzufügen. $b f i$ Education setzt sich deshalb für die Integration von moving image media education in anderen Fächern, vor allem Englisch, ein, als Problem bleibt jedoch, wie man die Qualität des Medienunterrichts sichert.

Der englische Lehrplan (National Curriculum) empfiehlt, dass Lehrer/innen Filme im Englischunterricht in den Klassen fünf bis acht (in England Altersstufen 11-14) analysieren. Leider sind Lehrer/innen, die es sich zutrauen moving image media $\mathrm{zu}$ unterrichten, immer noch in der Minderzahl. Die meisten Lehrer behandeln den Inhalt und Kontext des Films, ohne auf die Filmsprache oder die Konstruktion des Films einzugehen. In den höheren Klassen erfreut sich media studies zwar immer mehr Beliebtheit, wird aber trotzdem oft noch als „Randfach“ betrachtet. Basierend auf akademisch-kritischen Theorien setzen sich „media studies“ mit verschiedenen Interpretationen der Wirkungsweise von Medien auseinander. Dies geschieht nicht nur durch theoretische Analyse, sondern auch zunehmend mit eigenen Medienproduktionen, womit die Konstruktionen und Wirkungsweisen verschiedener Medien verdeutlicht werden können. ,Media studies': ein Wahlfach, das immer noch von weniger als 5\% der Schüler zwischen 14 und 19 Jahren belegt wird.

Generell ist der Stellenwert von Medienpädagogik in englischen Schulen und Lehrplänen zwar höher als in Deutschland, aber immer noch bei weitem nicht so anerkannt, wie er sein sollte. 


\section{Moving image media education in Grundschulen}

bfi Education ist der Ansicht, dass Kinder ein Grundrecht haben, über Bildmedien in der Schule zu lernen. Vor allem, da sie tagein tagaus mit Film und Fernsehen konfrontiert werden, oft in weitaus größerem Ausmaß als mit gedruckten Texten. Das bewegte Bild ist das Leitmedium des 20. Jahrhunderts, Fernsehen und Video gehören zu den ersten kulturellen Erfahrungen, die Kinder in der westlichen Welt im 21. Jahrhundert machen. Sie lernen schon im frühen Alter den flickernden Bildern auf dem Bildschirm Sinn zu entlocken, die Änderungen von Größen und Einstellungen, die Wechsel der Hintergründe und Charaktere, die Geräusche und Musiksequenzen, die entweder Gefahr, Komödie, Spannung oder ein Happy End signalisieren können, zu verstehen. Bereits im Alter von drei Jahren beherrschen die meisten Kinder nicht nur ihre Muttersprache, sondern haben sich zusätzlich Filmsprache angeeignet: die Codes und Konventionen, mit denen bewegte Bilder Geschichten erzählen. Obwohl Kinder in diesem Alter noch ein relativ limitiertes Verständnis der Filmsprache haben, - sie können oft noch nicht unterscheiden, was „Echt“ und was „Schein“ ist, und mögen relativ wenig von dem, was sie in den Nachrichten, Filmen, Dokumentationen oder Seifenopern sehen, wirklich verstehen -, haben die Meisten schon ihre Film- und Video- bzw. DVDFavoriten, die sie oft in und auswendig kennen. Lehrer/innen sind sich meist einig, dass es einfacher ist anzuerkennen und zu erweitern, was Kinder schon wissen und können, als von Null anzufangen. Und so ist nahe liegend und macht Sinn für Schulen, auf diesem Wissen aufzubauen, und vor allem den Enthusiasmus, den Kinder für Filme zeigen, zu nutzen.

\section{Look Again! Moving image media education als Wahrnehmungs- training}

Es macht Sinn moving image media education in existierende Fächer einzubauen und diese zu erweitern und $\mathrm{zu}$ variieren, ohne zusätzliche Arbeit für Lehrer zu schaffen oder ein neues Fach zu kreieren. Bildmedien ,sprechen' eine universelle und globale Sprache, und dies kann vor allem für Kinder, die mit Sprachbarrieren zu kämpfen haben, große Vorteile bringen. Das von $b f i$ Education veröffentlichte Unterrichtsheft Look Again führt detailliert aus, wie moving image media education in die Lernerfahrungen aller Drei- bis Elfjährigen eingebettet werden kann. Es wird erklärt, wie man mit acht verschiedenen Grundlehrtechniken moving image media unterrichten kann und wie man diese vor allem in den Sprach- und Schreibunterricht (literacy teaching) integrieren kann. Die direkte Verbindung mit dem Sprachunterricht ist dabei sehr wichtig, da diese die direkten Verbindungen, Parallelen, aber auch Unterschiede zum Lehren von Drucktexten demonstriert.

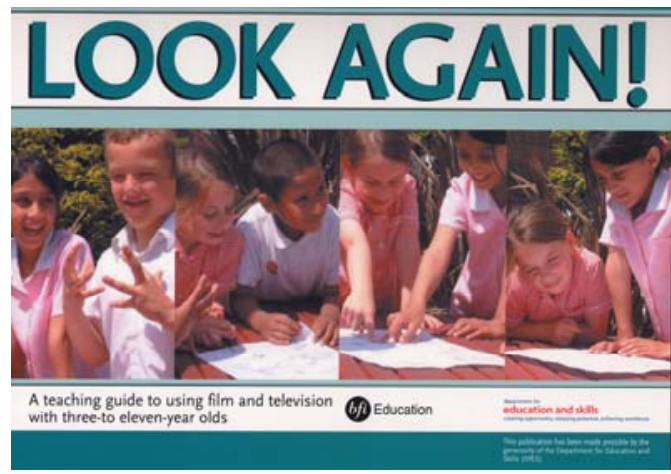

Unsere Argumentation, die auf solider wissenschaftlicher Forschungsarbeit beruht, haben wir in fünf grundlegende Argumente für moving image media education aufgeteilt. Ich habe versucht die Wichtigsten davon zusammenzufassen.

\section{Aktives Lernen: Vom Bekannten zum Unbekannten}

Viele Studien haben hervorgehoben, dass das Gehirn aktiv lernt. Mit anderen Worten, was auch immer gelehrt wird, der/die Lernende muss aktiv beteiligt sein, damit Bedeutung entstehen kann. In den tieferen Prozessen des Lernens bewegen sich Lernende vom Bekannten zum Unbekannten, dabei machen sie das Bekannte bewusst und erweitern dieses Wissen und verstehen dadurch, was sie schon wissen (Bruner 1986; Vygotsky 1978). Wenn sehr junge Kinder eingeschult werden, wenden Lehrer/innen dies an - sie arbeiten mit dem Bekannten und Gegebenen, um ihren Schülern zu helfen, ihre Umwelt zu verstehen (Rogoff 1992). Fast alle Kinder in unserer Kultur haben von klein auf extensive Erfahrungen mit Film, Fernsehen und Video (Kress 1997; Bromley 1999; Marsh and Hallet 1999). Es ist notwendig, mit genau den Texten anzufangen, die Kinder schon aktiv „lesen“, um Kindern zu helfen Texte zu „lesen“ und dabei vor allem die tieferen Ebenen zu verstehen und gleicher Maßen Texte selbst zu kreieren (Browne 1999; Meek 1991). Dieser Lernprozess verblasst nicht, wenn Kinder das Alphabet lernen. Im Gegenteil, er fördert ihre Fähigkeiten zu schreiben und zu lesen und Texte jeglicher Art, sowohl in der Schule als auch außerhalb, zu verstehen (Heath 1983. Meek 1988, Gregory \& Williams 2000).

\section{Das Verbinden von Freizeit und Schule}

Eine Reihe wissenschaftlicher Studien betonen, dass die erfolgreichsten Schulen und Lehrer/innen mit dem kulturellen Wissen und Verständnis arbeiten, das sich ihre Schüller außerhalb der Schule angeeignet haben. Medientexte spielen dabei eine tragende Rolle. Anstatt wie viele Grundschullehrer/innen, den Medieneinsatz als irrelevant oder gar schädlich zu betrachten („Kinder sehen viel zu viel fern in ihrer 
Freizeit"), teilen viele Experten die Meinung, dass Medienwissen in generelles Wissen umgesetzt werden kann. Dabei sollten AVMedien nicht nur als Unterhaltung, Belohnung oder als bloße „Hilfsmittel“ eingesetzt werden, sondern sollten ebenso als Texte anerkannt werden wie Druckmaterialien. Dies heißt auch, dass Schüler/innen die Möglichkeit haben sollten, selbst kreativ zu sein. Je mehr sie dabei über Konventionen und „Sprache“ gelernt haben, desto fähiger werden sie sein, ihre eigenen Texte zu kreieren.

\section{Medientexte und Filmtexte}

Look Again! versucht Lehrer/innen und Erzieher/innen vom Glauben abzubringen, dass Film und Fernsehen Kinder von der ,wirklichen' Bildung ablenken. Bunte Fantasien, graue Realität, Szenen von gestern und bewegte Bilder von der Jahrhundertwende, liveÜbertragungen aus der ganzen Welt. Manche dieser Bilder lügen, manche sind offensiv oder beängstigend, manche sind trügerisch und nutzlos. Das Gleiche gilt jedoch für Bücher. Wir lernen lesen, weil Bücher uns die einzigartige Möglichkeit geben, das Wissen und die Vorstellungskraft Anderer zu teilen. Aua genau demselben Grund sind alle Kinder berechtigt, über Bildmedien $\mathrm{zu}$ lernen und sich nicht nur privat mit ihnen auseinanderzusetzen. Sobald sie lernen, Medientexte kritisch zu lesen, werden sie auch in ihrer Freizeit bewusster mit Medien umgehen. Das heißt natürlich nicht, dass sie deshalb nicht trotzdem stundenlang obskure Animationsfilme anschauen, aber wir dürfen dabei nicht vergessen, dass auch diese ,gelesen“ werden wollen. Nicht alle Medientexte eignen sich für den Unterricht, aber das gilt auch für Bücher. Medientexte müssen genauso sorgfältig ausgesucht werden wie gedruckte Texte. Lehrer/innen müssen genauso wie Schüler/innen lernen, reiche Texte - inhaltlich, aber vor allem auch filmsprachlich - von minderwertigen Texten zu unterscheiden.

\section{Die Filmauswahl}

Bei der Filmauswahl ist es sehr wichtig altersgerechte Filme mit einem hohen Niveau nicht nur an Inhalt, sondern vor allem auch in Bezug auf die Filmsprache zu finden und dann Lehrer/innen und Schüler/innen beurteilen zu lassen, ob die Filme auch für den Unterricht geeignet sind. Leider sind oft sehr gute Filme mit Schimpfwörtern gespickt, die es Lehrer/innen schwer machen, den Film im Unterricht $\mathrm{zu}$ verwenden. Gleichzeitig sollte man jedoch auch die Schüler/innen nicht unterschätzen, die oft inhaltlich und film- sprachlich anspruchsvollere Filme bevorzugen und viel leichter über „unangemessene“ Sprache (im Unterrichtskontext) hinwegsehen.. Wenn Lehrer/innen Filmtexte im Unterricht verwenden, greifen sie oft auf familiäre und konventionelle Filme zurück - zum Teil weil Schüler einen direkteren Zugang zu diesen Filmen haben, zum Teil aber natürlich auch, weil konventionelle Filme einfacher zu bekommen sind.

bfi Education hat sich zur Aufgabe gemacht, Filme auszusuchen, die nicht nur auf Familiärem aufbauen, sondern vor allem auch Erwartungen in Frage stellen und die Filmerfahrungen von Schülern erweitern und ausbauen.

\section{Show us a Story}

Show us a Story ist eine Lehrhilfe, die sechzehn verschiedene Spielfilme und Animationsfilme für den Grundschulunterricht empfiehlt und Ideen und Richtlinien gibt, wie man mit ihnen und über sie lehrt. Die Filme wurden sorgfältig nach Filmsprache, Inhalt und Erhältlichkeit als VHS oder DVD ausgesucht und erleichtern Lehrer/innen die

\section{5.}

\section{Moving Images in the Class- room}

Moving Images in the Classroom ist eine Lehrhilfe um Film und Fernsehen in weiterführenden Schulen zu unterrichten. Es wird beschrieben, wie grundlegende Lehrtechniken und praktische Aktivitäten rund um Film und Fernsehen in verschiedenen Fächern wie Englisch, Geschichte, Kunst, Erdkunde usw. angewandt werden können. Argumente für und Erfahrungen über das Unterrichten mit Film und Fernsehen werden aufgeführt und Beispiele von Filmemachen mit digitalen Medien besprochen. Schulen in Großbritannien investieren mehr und mehr in Hardware, Software and Netzwerke. Dies führt nicht nur zu einfacherem Zugang zu Bildmedien, sondern fördert auf lange Sicht den kreativen Umgang mit Filmmedien, was nicht nur zum generellen Verständnis beiträgt, sondern auf lange Sicht einen ebenso hohen Stellenwert haben sollte wie Schreiben.

\section{Lehren mit Kurzfilmen}

Der Vorteil von Kurzfilmen ist, dass sie im Unterricht als ganzheitliche Texte behandelt werden können. Sie können ebenso problemlos in eine Unterrichtsstunde eingebaut werden wie Drucktexte. Mehrmaliges Anschauen, das nötig ist um Filme zu analysieren, bietet sich geradezu an bei einem Film, der nicht länger als fünfzehn Minuten ist, und ermöglicht den Schüler/innen das Zuschauerlebnis zu teilen. Lehrer/innen, die mit Film im Unterricht arbeiten, sind immer wieder von den vielen positiven Aspekten des Einsatzes von Kurzfilmen überrascht und erkennen den erhöhten Motivationsfaktor und die 
höhere Lerneffizienz durch diesen Einsatz an. Arbeit mit Kurzfilmen im Klassenzimmer schließt niemanden aus, vor allem nicht Schüler/innen mit Sprachbarrieren und motiviert oft zur Beteiligung am Unterricht. Dass Schüler/innen, die sonst eher auffallend ruhig waren, oft das Filmwissen und Sprachvermögen haben, um „mitreden“ zu können, haben viele Lehrer/innen erfahren, wenn sie Kurzfilme im Unterricht eingesetzt haben. Es gibt viele Filme, die für den Grundschulunterricht geeignet sind, die keinen gesprochenen Ton haben und die besonders Schüler/innen, die eine andere Muttersprache sprechen oder Lernschwierigkeiten haben, ermöglichen, aktiv und autonom zu lernen.

\section{Story Shorts und literacy}

Story Shorts war das erste Lernpaket von $b f i$ Education, das Filmerziehung in Grundschulen aktiv mit Kurzfilmen unterstützt, und wurde von Kollegen in $b f i$ Education mit der Hilfe von Grundschullehrer/innen entwickelt. Story Shorts ist eine Kombination einer Kurzfilm-Kompilation mit fünf Kurzfilmen und einem Begleitheft für den Unterricht in der Grundschule (Primary School) für Key Stage 2 - also für 7- bis 11-jährige Schüler. In einem fast zweijährigen Pilotprojekt/Versuchsmodell haben Kollegen mit verschiedenen Grundschulen zusammengearbeitet, um verschiedene Kurzfilme im Klassenzimmer auszuprobieren und gleichzeitig Feedback und Ideen von den Lehrer/innen zu bekommen. Das Projekt war gleich erfolgreich, und man einigte sich auf einen Kanon von fünf Kurzfilmen und eine Reihe von Unterrichtsaktivitäten, die dann in verschiedenen Schulen angewandt und verfeinert beziehungsweise abgeändert wurden. So ist das Begleitheft zwar von bfi Education geschrieben worden, beinhaltet jedoch viele Beiträge und Ideen von Lehrer/innen, was es sehr unterrichtsgerecht und spezifisch macht. Die fünf Filme, von drei bis 14 Minuten lang, repräsentieren eine Auswahl unterschiedlicher filmischer und erzählerischer Ausdrucksarten. Vier Filme sind Animationsfilme und ein Kurzspielfilm; zwei Filme haben Dialog, zwei nur Musik und ein Film hat nur natürliche Geräuscheffekte; drei Filme sind Erzählungen mit einem Anfang, einer Mitte und einem Ende, und zwei sind eher abstrakt und impressionistisch. Wir wollen vor allem Lehrer/innen zeigen, wie man Schülern die Konventionen von Filmkonstruktionen bewusst macht und gleichzeitig ihre Lese- und Schreibfähigkeiten (literacy) erweitert. Wie es

David Parker (2002, S. 40) formuliert hat: „Das Feedback von Lehrer/innen, die mit Story Shorts über einen längeren Zeitraum hinweg gearbeitet haben, unterstützen unsere Hypothese, dass es tiefgehende Verbindungen zwischen Erzählstrukturen in Texten und Filmen gibt, die sich geradezu für Schreibaufgaben anbieten. Filme können, wenn sie von Kindern genauestens als Texte im Unterricht gelesen werden, als eine Art Gerüst funktionieren."

Colette Higgins, eine der Lehrer/innen, die am Story Shorts Projekt mitgearbeitet hat, war positiv von ihren Schüler/innen überrascht: „Alle Schüler/innen haben die Prinzipien der Filmsprache sehr schnell begriffen und in ihren Wortschatz eingebaut. Sie konnten diese nicht nur in Texten analysieren, sondern waren fähig, Parallelen in ihrem Schreiben zu erzeugen."

Dazu ein paar Auszüge aus den Aufsätzen der Schüler/innen.

\section{Die Augen der Leser nach oben richtend:}

Die Kirchenglocken hoch oben im Turm kündigten laut einen neuen Tag an.

\section{Story Shorts}
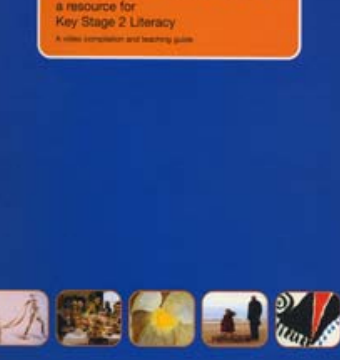

Die Vögel flogen in perfekter Formation über den Kirchturm.

Die Augen der Leser nach unten richtend:

Der Balancierstab fiel aus seinen Händen in die Tiefe der Schlucht.

Kleine Details hervorbebend:

Seine Uhr war an einer kleinen goldenen Brosche in der Form eines Medaillons befestigt.

In der Mitte war ein silberner $\mathrm{Ba}$ lancierstab der die Eingravierung „El Caminante“ trug.

Higgins listet noch viel mehr Beispiele auf, die zeigen, wie sehr das Studieren des Films El Caminante das Schreiben ihrer Schüler/innen beeinflusste. Innerhalb einer Unterrichtswoche sahen die Schüler den Film mehrmals an, um die Szenerie, die Erzählstruktur, Anfang und Ende, die Entstehung von Spannung und die Entwicklung der Charaktere zu erkunden. Wie die Auszüge schon demonstriert haben, bereicherte dieser Prozess das Schreiben der Schüler/innen und half ihnen, beim

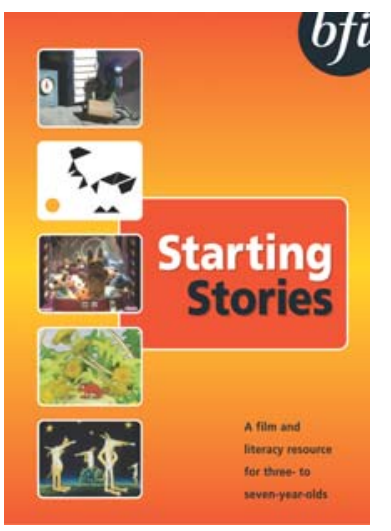

Schreiben bewusst spezifische Effekte zu erzeugen.

\section{Starting Stories}

Da Story Shorts solch ein Erfolg für Lehrer/innen und Schüler/innen war, haben wir bald danach ein Lehrpaket für Drei- bis Siebenjährige nach dem gleichen Konzept entwickelt: Fünf verschiedene Animationskurzfilme auf VHS oder DVD und ein Begleitheft mit viel Hintergrundinformationen und Aktivitäten. 


\section{Screening Shorts}

Screening Shorts, ein Lehrpaket für elf- bis vierzehnjährige Schüler/innen, ist eine Kurzfilmkompilation mit sechs Kurzfilmen und drei Werbefilmen und einem Begleitheft in CDROM-Format.

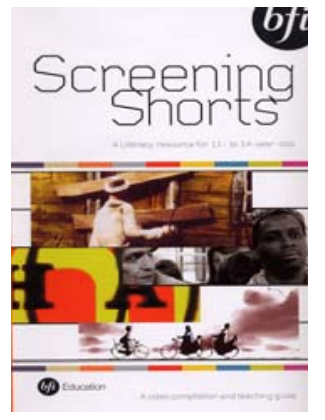

\section{Kurzfilme im Klassenzimmer}

Ich könnte viele Beispiele für den Einsatz von Kurzfilmen im Klassenzimmer beschreiben, beschränke mich aber auf drei kurze „Case Studies".

\section{Case Study 1}

Eine Gruppe von Kindergartenkindern sehen sich zusammen Andersartig von Christina Schindler (Starting Stories Kompilation) an. Dieser Film handelt von einem jungen Chamäleon, das seine Farbe nicht wechseln kann. Die Kindergartenlehrerin befragt die Kinder über die Verstecke und Gefühle des Chamäleons und über die Farben, die es nicht annehmen kann. Die Kinder denken sich dann ihre eigenen Szenen aus. Sie malen das Chamäleon in neuen Situationen und Hintergründen. Die Kindergartenlehrerin hilft daraus ein Buch mit einer neuen Geschichte zu machen.

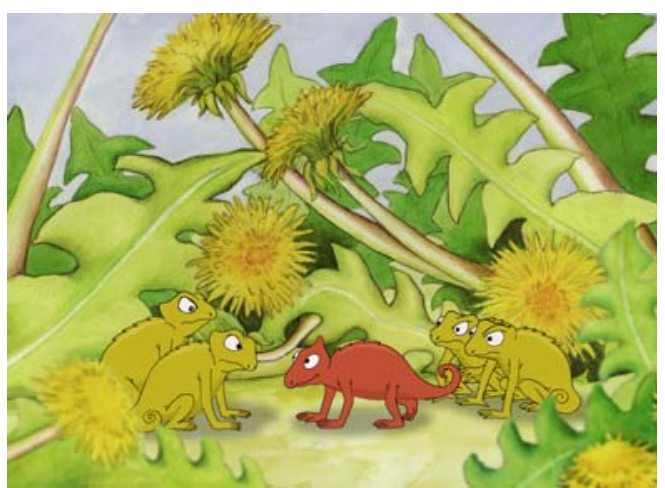

Andersartig

(Christina Schindler, Deutschland, 2002)

\section{Case Study 2}

Eine Klasse von Neun- und Zehnjährigen schaut sich den Anfang des Kurzfilms Mavis and the Mermaid (Story Shorts Kompilation) an. Sie diskutieren über die Wichtigkeit des Anfangs und des Endes einer Geschichte. Sie konzentrieren sich dann darauf, wie die Eröff- nungssequenz des Films die Information vermittelt, die der Zuschauer braucht um den Film zu verstehen. In Gruppenarbeit gehen sie dann auf Details der Titelgrafiken, des Soundtracks, der Belichtung, Farben und Kameraeinstellungen ein.

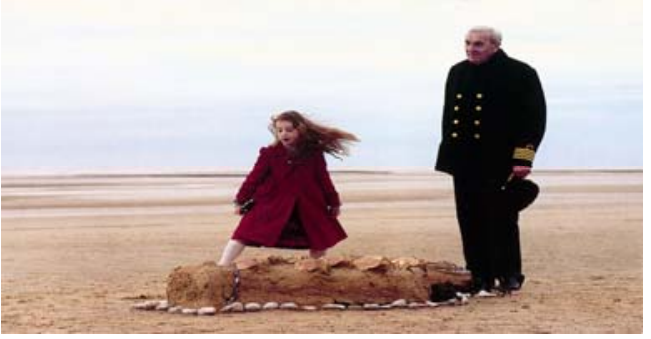

Mavis and the Mermaid

(Juliet McKoen, UK, 2000)

\section{Case Study 3}

Eine Klasse von Dreizehn- bis Vierzehnjährigen schaut sich den Kurzfilm Father and Daughter von Michael Dudok de Witt an. Sie erzählen die Geschichte in verschiedenen Erzählperspektiven nach. Dann kreieren sie ihr eigenes Storyboard und recherchieren, wie sie daraus ihren eigenen Animationsfilm machen können.

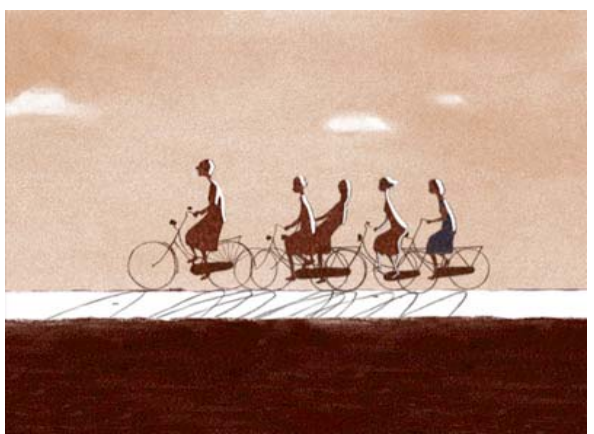

Father and Daughter

(Michael Dudok de Wit, UK, 2000)

Die Möglichkeiten sind so vielfältig, dass wir immer wieder positiv überrascht sind, was sich Lehrer/innen und Schüler/innen alles einfallen lassen. Idealerweise führen diese Aktivitäten auch zum kreativen Einsatz mit Film. Filmemachen, egal ob Spielfilm, Dokumentar- oder Animationsfilm, bietet Schülern eine andere Möglichkeit ihre Texte bewusst zu gestalten. Kostengünstige digitale Videotechnik gibt Schulen immer mehr Möglichkeiten, Filmemachen in den Unterricht einzubauen. Wie auch nur belesene Autoren gute Schriftsteller machen, erfordert gutes Filmemachen auch qualifizierte Zuschauer.

Weitere Informationen über $b f i$ Education: www.bfi.org.uk/education 


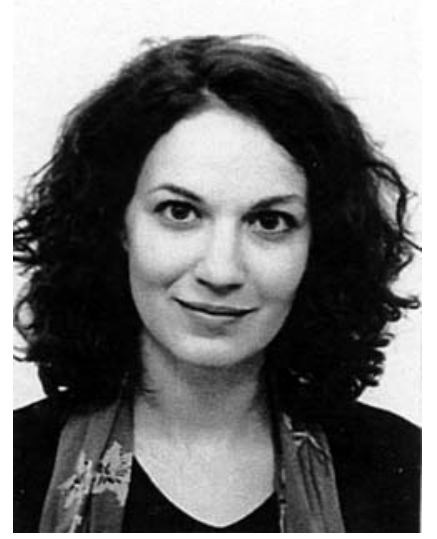

\section{Caren Willig}

ist seit über drei Jahren Project Manager für Unterrichtsmaterialien in der Abteilung Education des British Film Institute. In dieser Funktion recherchiert und produziert sie Lehrmaterialien für den Unterricht, hauptsächlich Video- und DVD-Kompilationen, aber auch Lehrbücher und online-Lehrhilfen.

Nach dem Studium der Rhetorik und der Literatur- und Kulturwissenschaft in Tübingen und Hildesheim hat sie ihren Master in Media and Communications am Goldsmiths College in London absolviert. Die Anstellung am British Film Institute vor sechs Jahren ermöglichte ihr, in London zu bleiben und - wider Erwarten vieler Verwandten - Geld zu verdienen. 